\title{
Asymmetric belief sensitivity and justification explain the Wells Effect
}

\author{
N. Ángel Pinillos (pinillos@asu.edu) \\ School of Historical Philosophical and Religious Studies, Arizona State University \\ Phoenix, AZ, USA \\ Sara Jaramillo (sdjarami@asu.edu) \\ School of Social and Behavioral Sciences, Arizona State University \\ Phoenix, AZ, USA \\ Zachary Horne (Zachary.Horne@asu.edu) \\ School of Social and Behavioral Sciences, Arizona State University \\ Phoenix, AZ, USA
}

\begin{abstract}
Wells (1992) found that jurors are more likely to find a defendant guilty when the evidence against them is 'specific' (that is, when the evidence provides a causal mechanism for how an event occurred) as opposed to being based on base-rate information, or what Wells calls 'general' evidence. Enoch, Spectre, and Fisher (2012) propose that this epistemic difference can be explained by the "sensitivity" of beliefs formed on the basis of these two types of evidence where sensitivity is understood as a counterfactual condition on knowledge judgments. They argue that beliefs are sensitive when formed on the basis of specific evidence, but not when they are formed on the basis of general evidence. In two preregistered experiments, we tested this hypothesis. We replicated an earlier finding that specific, as opposed to general evidence, is more likely to lead to knowledge judgments. Consistent with the hypothesis of Enoch and colleagues, we also found that sensitivity partially mediates the relationship between evidence type and knowledge attributions.
\end{abstract}

Keywords: Wells effect; counterfactual reasoning; knowledge sensitivity; open science

\section{Introduction}

Imagine that you are a juror deliberating on a civil suit against the Blue bus company which is being accused of running over a dog during one of its routes. The only evidence that is presented to you is that a commercial bus killed a dog and that $80 \%$ of the commercial buses in the area are run by the Blue bus company. Is the Blue bus company guilty of killing the dog? According to Wells (1992), most people do not think you should convict on this evidence. Now suppose instead that the only evidence presented to you is that a witness reports seeing a Blue bus run over the dog and that this witness is $80 \%$ reliable. In this case, is the evidence sufficient for finding the Blue bus company guilty of killing the dog? According to Wells (1992), people are more likely to find a defendant guilty when the second kind of evidence, termed specific evidence, is given than when the first kind of evidence is given (termed "general" evidence). Strikingly, people exhibit this pattern of judgments even though they also perceive the probability of guilt as being the same in both cases. The tendency to trust specific more than general evidence is also reflected in the law. As Enoch and Fisher (2015) report, "Courts and legal scholars often view [general evidence] with suspicion, treating it as inadmissible even when it is probabilistically equivalent to individualized [specific] evidence." The explanation of the Wells effect is therefore not only important to understanding how people reason about evidence but is also pertinent to the law.

What explains the Wells effect? One possibility is that in the first case, the evidence is purely general in the sense that it is not causally connected to the actual killing of the dog: the fact that $80 \%$ of the buses are operated by the Blue bus company is, in a sense, independent of the actual killing of the dog. In contrast, the evidence in the second case is specific to the case; there is a causal connection between a witness reporting the Blue Bus company killed the dog and the dog being killed. Consistent with this possible explanation, empirical accounts on the Wells Effect often focus on counterfactual thinking - the ability to consider what could have happened but did not happen. For example, Niedermeier, Horowitz, \& Kerr (1999) and Sykes and Johnson (1999) account for the reluctance to find the defendant guilty in general cases by appealing to the ease in which the possibility that the defendant is innocent comes to mind. Along these lines, Enoch et al. (2012) proposed that general evidence is considered weaker than specific evidence because beliefs formed on the basis of the former, but not the latter, are sensitive (a term which we explain below). The main goal of this paper is to test this hypothesis.

Many philosophers think that the notion of sensitive belief can explain a wide range of judgments similar to the Wells Effect (Black \& Murphy, 2007; DeRose, 1999; Dretske, 1981; Ichikawa, 2011; Nozick, 1981; Roush, 2005). Philosophers typically define a 'sensitive belief' as follows:

An agent's belief that $P$ is 'sensitive' just in case If $P$ were false, then the agent would no longer believe $P$.

For example, your belief that you are reading a paper right now is sensitive. This is because if you weren't reading a paper, you would be making coffee, let us suppose, and so would no longer believe you were reading a paper. Sensitive beliefs are counterfactually robust in the sense that they "track" the facts in possible circumstances. This is why 
some philosophers have held that if an agent knows $P$, then her belief that $P$ has to be sensitive. Let's call this thesis itself 'sensitivity'. Sensitivity is a type of counterfactual condition on knowledge.

Take another example: Consider a person who believes that his beloved pet is healthy, not through good evidence, but because of wishful thinking. We can suppose further that his pet is in fact healthy. Our intuitive reaction is that this agent does not really know his pet is healthy. But why doesn't the agent know this? According to the sensitivity account, the reason is that this agent's belief is not sensitive. For example, although his pet is actually healthy, if the pet were sick, the wishful-thinking agent would continue to think that the pet was healthy. Because his belief is not properly tracking the facts in the world, he doesn't really know his pet is healthy. In contrast, suppose the agent believes his pet is healthy, not through wishful thinking, but because he got a good report from a reliable vet. In this case, his belief is more likely to be sensitive (and hence truth tracking) because if his pet were sick, he would believe that his pet was sick. This is because if his pet were sick, the reliable vet would have told him so.

These two examples demonstrate how the sensitivity hypothesis is supposed to account for our epistemic intuitions. For our purposes, what is important is that sensitivity gives us a counterfactual condition on the perceived strength of one's evidence. We know that counterfactual reasoning also plays a role in a number of other cognitive phenomena. It has been implicated in planning and prediction (Barbey \& Sloman, 2007; Epstude \& Roese, 2008; Markman, McMullen, \& Elizaga, 2008; Roese, 1999; Smallman \& Roese, 2009; Tobia, Guo, Schwarze, Boehmer, Gläsher, Finckh, \& Sommer, 2014), generating emotions (Alicke, Buckingham, Zell, \& Davis, 2008; Brassen, Gamer, Peters, Gluth, \& Buchel, 2012; Coricelli \& Rustichini, 2010; Davis, Lehman, Wortman, Silver, \& Thompson, 1995; Miller, Markman, Wagner, \& Hunt, 2013; Pieters \& Zeelenberg, 2005; Roese \& Olson, 1997, 2007), learning (Byrne, 1997; Epstude \& Roese, 2008; Smallman \& McCulloch, 2012), as well as in moral and causal reasoning (Halpern \& Hitchcock, 2015; Malle, Guglielmo, \& Monroe, 2014). Although counterfactual reasoning has been found to play a large role in thinking across many psychological domains, we are not aware of any experimental work directly examining how counterfactual judgments, such as those involved in the definition of sensitivity, relate to attributions of knowledge. This is notable because, as discussed, counterfactual reasoning is prominently featured in theories of knowledge attributions in the philosophical literature.

In the present studies, we investigated whether people's judgments about knowledge can be explained by the sensitivity hypothesis. Specifically, we sought to test this sensitivity account as an explanation for the Wells effect, and related effects central to ongoing debates in epistemology (Friedman \& Turri, 2014). The original Wells effect concerned judgments about whether a defendant should be found guilty. Following Wells and our discussion so far, our approach is to investigate not determinations of guilt, but attributions of knowledge. We predicted that people differentiate general and specific evidence because of their differential sensitivity-beliefs formed on general evidence are less sensitive than beliefs formed on specific evidence.

\section{General Methods}

Analytic Approach To test our hypotheses, we performed Bayesian mixed-effects modeling using the R package brms (Bürkner, 2018). We set regularizing priors for all population-level effects in our models, which we detail below. These priors are recommended because they provide conservative effect size estimates and reduce the likelihood of overfitting (Gelman, Lee, \& Guo, 2015; McElreath, 2016). Following the recommendations of Liddell \& Kruschke (2018), Likert data were modeled with a cumulative probability distribution. The cumulative distribution is recommended for Likert scale data because it assumes that ordered responses represent a continuous latent construct (in this case, the tendency to attribute knowledge to an agent).

Preregistration We preregistered the data collection plan, analyses, and predictions for both experiments. Experimental scripts, analyses, and supplementary online materials are available on the Open Science Framework at https://osf.io/pw7s8/.

\section{Experiment 1}

Participants We powered our study to detect a Cohen's d of .2 for a two-condition within-subjects design with $80 \%$ power. To this end, 201 participants were recruited through Amazon's Mechanical Turk (43\% women, $M_{\text {age }}=36$ years old). Participants were paid $\$ 0.50$ for participating in the study. Participants were excluded for missing questions checking their attention (e.g., "Select perhaps knows from the options below"). Our exclusion criteria were determined a priori and were in accordance with our study preregistration. After excluding participants who missed questions checking their attention, 170 participants remained in our sample.

Procedure In Experiment 1, we examined whether the Wells effect can be explained by the sensitivity hypothesis. To test this, we randomly presented participants with six scenarios in a within-subjects design (three topics $\times$ two conditions $=$ six vignettes) adapted from Friedman and Turri (2014). In these scenarios, a protagonist is described as researching a question in which they rely on either general, base-rate information or specific, mechanistic information to draw their conclusion. For example, participants considered the following scenarios:

General: Bob wonders if his spider plant contains the chemical aracnium. He consults a very reliable book on 
spider plants. The book says that [98 or $99 \%$ randomly presented] of spider plants contain aracnium. So Bob concludes that his spider plant contains aracnium. And he is right: it contains aracnium.

Specific: Joseph wonders if his stinkwood flower will grow yellow stalks. He conducts a very reliable DNA test on the flower. The test shows that it is [98 or $99 \%$ randomly presented] likely that the stinkwood flower will grow yellow stalks. So Joseph concludes that his stinkwood flower will grow yellow stalks. And he is right: the flower grows yellow stalks

After reading each scenario, participants were asked on a four-point Likert scale whether, for example, "Bob knows his spider plant contains aracnium" $(1=$ Definitely does not [know], 4 = Definitely does [know]). After answering this question, participants then answered a question aimed at assessing the sensitivity of the protagonist's belief:

"If Bob's spider plant hadn't contained the chemical aracnium, what would Bob have thought after checking with the book on spider plants?"

Participants then judged whether "Bob would [would not] have thought that his plant contains the chemical aracnium." If participants agreed that Bob would have thought his plant contains aranium regardless, this would mean participants thought that Bob's belief was not sensitive. If they thought that Bob would not have thought that, then this would indicate participants thought that Bob's belief was sensitive.

Predictions We predicted that knowledge judgments would vary based on the condition participants read. Namely, we predicted that participants would be more likely to attribute knowledge to protagonists that formed their belief on the basis of specific rather than general information. In addition, we predicted that participants would think that a protagonist's belief in the General condition is not sensitive (relative to the Specific condition) and this would in turn lead to reduced knowledge attributions.

\section{Results and Discussion}

To test our first hypothesis, we performed ordinal mixed-effects modeling. This model regressed knowledge judgments on condition (Reference $=$ General condition) and included two group-level effects: (1) A group-level effect on vignette that allowed for heterogeneity in vignette intercept, (2) a group-level effect on Subject, which allowed for heterogeneity in both the slope and intercept of the condition effect on knowledge judgments. The model is specified below in brms syntax:

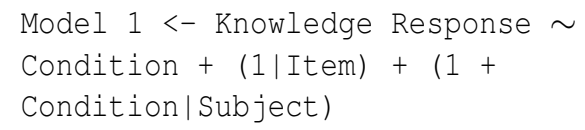

Bayesian analyses formulate model parameters as probability distributions wherein the posterior distribution

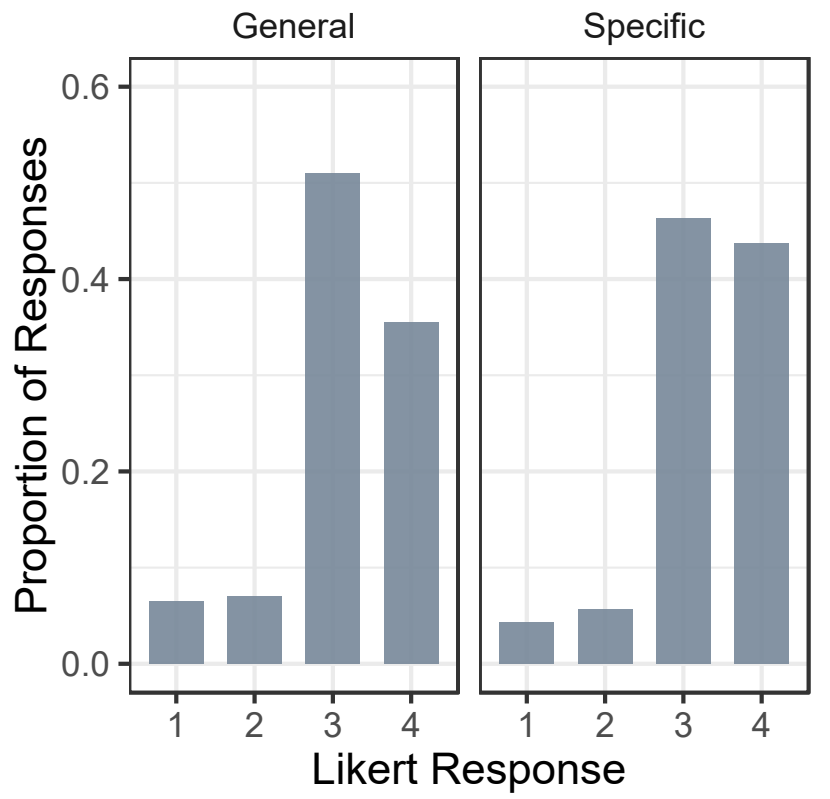

Figure 1: A histogram of the proportion of responses at a given Likert scale point (e.g., 1 = Definitely does not know; 4 $=$ Definitely knows) in the General and Specific conditions in Experiment 1 . The figure indicates that participants were less likely to attribute knowledge in the General condition than the Specific condition.

for a parameter $\theta$ is computed via the prior and the likelihood of $\theta$. To model the joint probability distribution of participants' knowledge responses, we specified the following priors over the possible effects each parameter could have on the response variable:

Experiment 1 - Model 1 Priors:

$$
\begin{aligned}
& \beta_{\text {Intercept }[1]} \sim \mathcal{N}(-1.73,1) \\
& \beta_{\text {Intercept }[2]} \sim \mathcal{N}(-.61,1) \\
& \beta_{\text {Intercept }[3]} \sim \mathcal{N}(.84,1) \\
& \beta_{\text {Condition }} \sim \mathcal{N}(0, .5) \\
& \Omega_{k} \sim \operatorname{LJJ}(1) \text { where } \Omega_{k} \text { is a correlation matrix of } \\
& \text { group-level parameters } \\
& \text { Group-level parameters were distributed as } \mathcal{N}(1,1)
\end{aligned}
$$

We predicted that participants would be more likely to attribute knowledge in the Specific condition than the General condition. This is what we observed, $b=0.72,95 \%$ CI $[0.38,1.05]$. Figure 1 indicates that in the General condition, the third Likert scale point [Perhaps knows] was the most probable response. In contrast, in the Specific condition both 3 [Perhaps knows] and 4 [Definitely knows] were probable responses, indicating that participants were more likely to attribute knowledge to a protagonist when the means by which the protagonist formed their belief was specific rather than general, as had been previously found (Friedman \& Turri, 2014).

What explains people's tendency to differentially ascribe 
knowledge when the probabilities in both situations are perceived as being similar? Our hypothesis is that this effect may be at least partially explained by sensitivity. That is, we predicted that people would think that an agent's beliefs in the Specific condition would be more sensitive than in the General condition. To test this prediction, we first performed logistic mixed-effects modeling. This model regressed participants' judgments of belief sensitivity (i.e., $1=$ Protagonist would still believe, $0=$ Protagonist would not still believe) on condition.

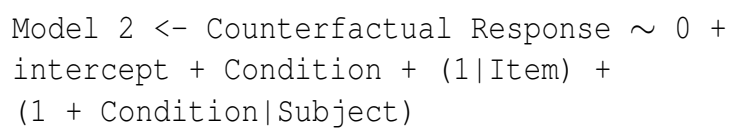

Experiment 1 - Model 2 Priors:

$$
\begin{aligned}
& \beta_{0} \sim \mathcal{N}(.75, .25) \\
& \beta_{\text {Condition } \sim \mathcal{N}(0, .5)} \\
& \Omega_{k} \sim \operatorname{LKJ}(1) \\
& \text { Group-level parameters were distributed as } \mathcal{N}(1,1)
\end{aligned}
$$

Here too, we observed the predicted effect of condition on participants assessments of belief sensitivity, $b=-0.60,95 \%$ CI [-0.94, -0.25] (see Figure 2).

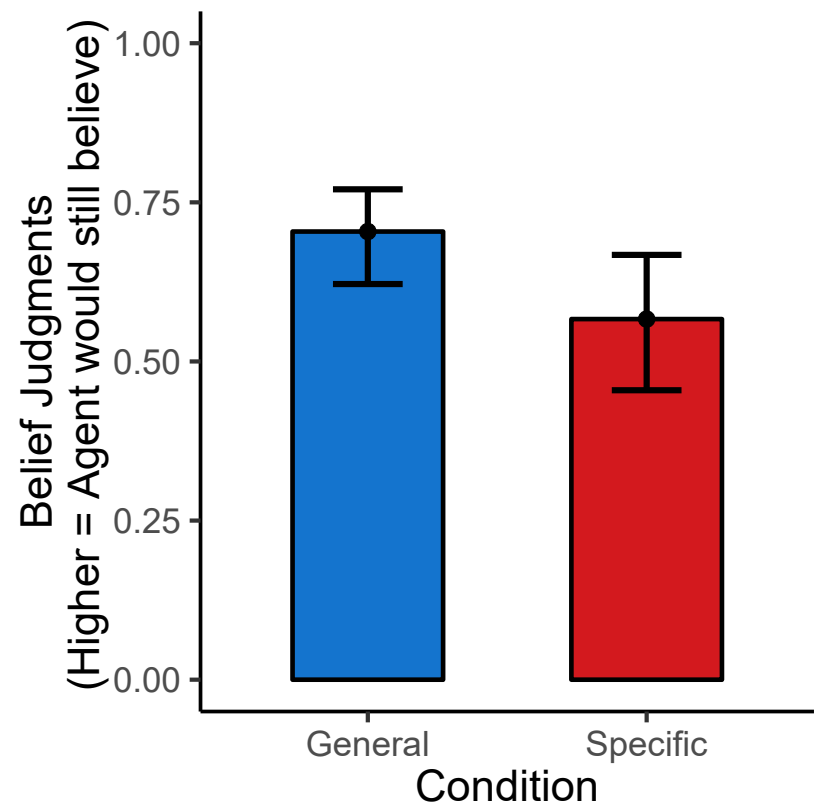

Figure 2: A marginal effects plot of belief sensitivity in the General and Specific conditions in Experiment 1. Error bars represent 95\% Credible Intervals (see Cummings, 2005 for interpreting within-subjects $95 \%$ CIs).

Our hypothesis was that people tend to differentially attribute knowledge in General and Specific conditions because of the sensitivity of the protagonists' beliefs in these two situations. We observed this effect. We followed up on this observation by performing mixed-effects mediation modeling to test whether sensitivity mediated the relationship between condition and knowledge attributions, as suggested by the partial correlations.

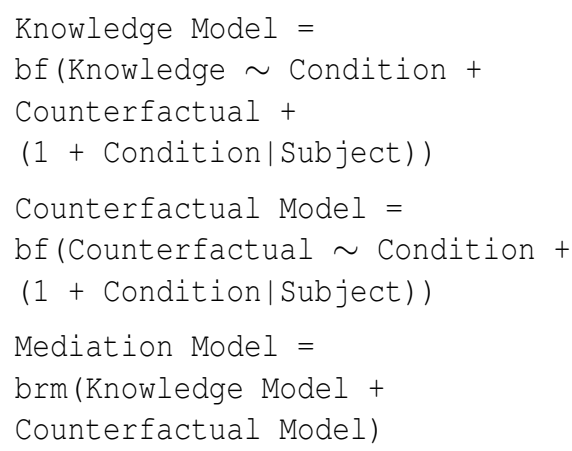

Mediation Model Priors:

$$
\begin{aligned}
& \text { All } \beta \sim \mathcal{N}(0, .5) \\
& \Omega_{k} \sim \operatorname{LKJ}(1) \\
& \text { Intercept and group-level parameters were } \\
& \text { distributed as } t(3,0,10)
\end{aligned}
$$

Consistent with our hypothesis, we observed the predicted mediation, $a b$ path $=.37,95 \%$ CI $[.08, .81]$.

\section{Experiment 2}

Experiment 1 provides preliminary evidence for the hypothesis that sensitivity can account for people being less likely to attribute knowledge in general versus specific cases. However, one possibility is that people think the agents in these two situations are not equally justified in drawing their conclusions and, further, that belief sensitivity is correlated with attributions of justification. Although it is often assumed that people are equally justified in believing that $P$ in both general and specific cases, this assumption has not been empirically tested. Consequently, we sought to rule out this alternative explanation of the results of Experiment 1 because justification is likely one of the most strongly predictive factors of knowledge attributions. To address this possibility, Experiment 2 directly replicated Experiment 1 but also included a measure of justification.

Participants We powered our study to detect a Cohen's d of .15 for a two-condition within-subjects design with $80 \%$ power. We anticipated the effect of counterfactual responses on knowledge attributions would be smaller after accounting for differences in justification between general and specific cases. A total of 344 participants were recruited through Amazon's Mechanical Turk (46\% women, $M_{\text {age }}=36$ years old). Participants were paid $\$ 0.50$ for participating in the study. After excluding participants who missed questions checking their attention, 288 participants remained in our sample. Our sample size and exclusion criteria were determined a priori and were in accordance with our study preregistration.

Procedure and Predictions The procedure of Experiment 2 was identical to that of Experiment 1. However, in Experiment 2, participants answered additional questions 
testing whether they thought the protagonist's beliefs were justified. For example, after reading each vignette participants also judged whether Bob was justified in concluding that his plant contains aracnium. Complete materials can be found in Table S4 in the SOM.

As in Experiment 1, we predicted that knowledge and sensitivity judgments would differ in the General and Specific conditions, and that sensitivity would mediate the relationship between condition and knowledge attributions. However, Experiment 2 also allowed us to test whether participants thought that (1) the protagonists in the Specific condition were more justified in drawing their conclusions and (2) rule out the possibility that justification alone rather than belief sensitivity accounts for the differential attribution of knowledge attributions in the General and Specific conditions.

\section{Results and Discussion}

As in Experiment 1, we first examined how condition (General vs Specific) affected knowledge and sensitivity judgments. We performed the same analytic procedure as in Experiment 1 . These analyses again revealed that both knowledge and sensitivity were predicted by whether the case participants were reading was general or specific, Knowledge: $\quad b=1.10,95 \%$ CI $[0.81,1.41)$ and Counterfactual: $b=-.52,95 \%$ CI $[-0.86,-.18$ ) (see Figures 3 and 4), along with the mediation of knowledge judgments by sensitivity $a b$ path $=.27,95 \%$ CI $[.05, .60)$. We then tested whether participants differentially attributed justification to the protagonists in the General and Specific conditions. We found that, indeed, participants judged that the protagonist in the Specific condition was more justified in drawing their conclusion than in the General condition, $b$ $=.86,95 \%$ CI $[0.51,1.20]$. Does justification, rather than sensitivity, account for the difference in knowledge attributions in general and specific cases? To examine this possibility, we performed ordinal mixed-effects modeling regressing knowledge attributions on justification and counterfactual judgments. Justification responses were treated as monotonic effects, following the recommendations of Bürkner and Charpentier (2018):

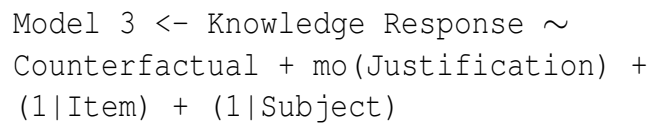

Experiment 2 - Model 3 Priors

$$
\begin{aligned}
& \beta_{\text {Intercept }[1]} \sim \mathcal{N}(-2,1) \\
& \beta_{\text {Intercept }[2]} \sim \mathcal{N}(-1.5,1) \\
& \beta_{\text {Intercept }[3]} \sim \mathcal{N}(4,1) \\
& \beta_{\text {Counterfactual }} \sim \mathcal{N}(0, .5) \\
& \beta_{\text {Justification }} \sim \mathcal{N}(2,4)
\end{aligned}
$$$$
\text { Group-level parameters were distributed as } \mathcal{N}(1,1)
$$

This analysis revealed that sensitivity accounted for unique variance in knowledge attributions over and above attributions of justification, $b=-0.51,95 \%$ CI $[-0.83$,

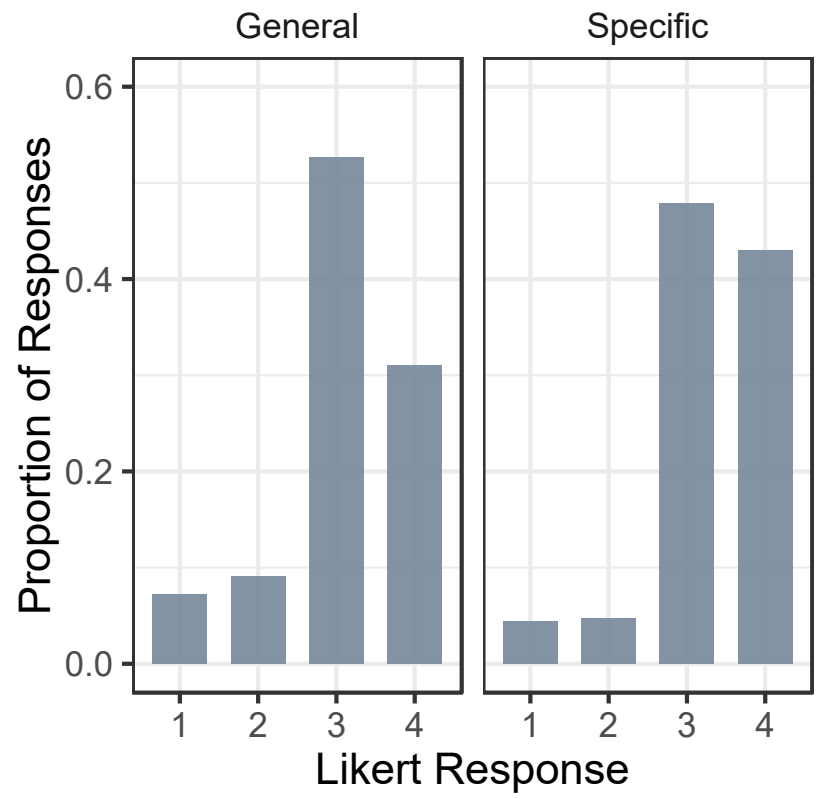

Figure 3: A histogram of the proportion of responses at a given Likert scale point (e.g., 1 = Definitely does not know; 4 $=$ Definitely knows) in the General and Specific conditions in Experiment 2. The figure indicates that participants were less likely to attribute knowledge in the General condition.

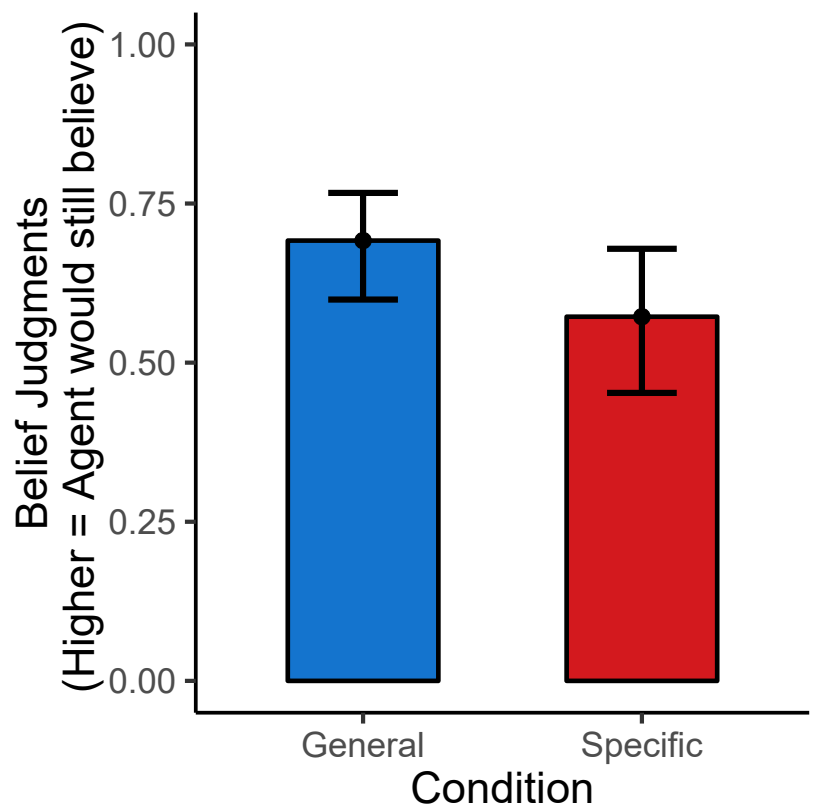

Figure 4: A marginal effects plot of belief sensitivity in the General and Specific conditions in Experiment 2. Error bars represent $95 \%$ Credible Intervals.

-0.19]. Thus, Experiment 2 provides evidence for two further conclusions: First, knowledge attributions likely 
differ in general and specific cases because of a difference in the perceived justification of agents in these situations. Second, and consistent with our prediction, people judge that beliefs formed on the basis of general evidence are insensitive (relatively) compared to beliefs formed on the basis of specific evidence, an effect that holds over and above the effect of justification.

\section{Discussion}

In psychology and the law, researchers have observed that jurors are less likely to trust general as opposed to specific evidence (Wells, 1992). However, this effect appears to extend beyond the courtroom: We found that knowledge attributions were similarly affected by manipulating whether evidence was general or specific, replicating earlier work that third-person knowledge attributions increase when the evidence available to the protagonist is specific as opposed to general. It is notable that this effect was found in cases that have little to do with the law or with witnesses, thus suggesting that the Wells effect is an instance of a more general phenomenon that goes beyond cases of witnesses or eyewitness memory specifically.

What explains people's tendency to distinguish general and specific evidence, given that the probabilities are fixed? We explored the hypothesis that general and specific evidence differ along more lines than their probabilities: namely, we examined whether sensitivity (a counterfactual condition) can account for the tendency to attribute knowledge in specific compared to general cases. Experiment 1 provided evidence that what explains the difference in knowledge judgments (across general and specific cases) is the perceived sensitivity of the belief at issue. In Experiment 2, we considered the alternative hypothesis that justification rather than sensitivity accounts for the difference in people's knowledge attributions in general and specific cases. We found that assessments of sensitivity account for variance in knowledge attributions over and above justification, although justification also differed by condition.

These experiments constitute the first empirical investigation demonstrating a link between knowledge attributions and sensitivity among non-philosophers. Further, this is the first piece of experimental evidence which suggests that a well-known effect in legal decision making - the Wells effect-can be understood in terms of epistemic theories that highlight the link between knowledge attributions and counterfactuals. Still, questions remain about the ability of the sensitivity hypothesis to account for other core epistemic phenomena. Indeed, a number of philosophers, for example, Williamson (2000), Blome-Tillmann (2015), and Hawthorne (2003), have pointed out that the sensitivity hypothesis cannot fully explain a range of intuitions regarding knowledge attributions. Consequently, subsequent experimental work should investigate these proposals to establish the scope and limits of the sensitivity hypothesis.

\section{References}

Alicke, M., Buckingham, J., Zell, E., \& Davis, T. (2008). Culpable control and counterfactual reasoning in the psychology of blame. Personality and Social Psychology Bulletin, 34, 1371-1381.

Barbey, A., \& Sloman, S. (2007). Base-rate respect: From ecological rationality to dual processes. Behavioral and Brain Sciences, 30, 241-254.

Black, T., \& Murphy, P. (2007). In Defense of Sensitivity. Synthese, 154, 53-71.

Blome-Tillmann, M. (2015). Sensitivity, Causality, and Statistical Evidence in Courts of Law. Thought: A Journal of Philosophy, 4, 102-112.

Brassen, S., Gamer, M., Peters, J., Gluth, S., \& Büchel, C. (2012). Don't look back in anger! Responsiveness to missed chances in successful and nonsuccessful aging. Science, 336, 612-614.

Bürkner, P. (2018). Advanced Bayesian Multilevel Modeling with the R Package brms. The R Journal, 10, 395-411.

Bürkner, P., \& Charpentier, E. (2018). Monotonic Effects: A Principled Approach for Including Ordinal Predictors in Regression Models. PsyArXiv.

Byrne, R. (1997). Cognitive processes in counterfactual thinking about what might have been. The psychology of learning and motivation: Advances in research and theory, 37, 105-154.

Coricelli, G., \& Rushtichini, A. (2010). Counterfactual thinking and emotions: regret and envy learning. Philosophical Transactions of the Royal Society B: Biological Sciences, 365, 241-247.

Cumming, G., \& Finch, S. (2005). Inference by eye: confidence intervals and how to read pictures of data. American Psychologist, 60, 170.

Davis, C., Lehman, D., Wortman, C., Silver, R., \& Thompson, S. (1995). The undoing of traumatic life events. Personality and Social Psychology, 21, 109-124.

DeRose, K. (1999). Contextualism: An Explanation and Defense. The Blackwell Guide to Epistemology, 185-203.

Dretske, F. (1981). The Pragmatic Dimension of Knowledge. Philosophical Studies: An International Journal for Philsophy in the Analytic Tradition, 40, 363 - 378.

Enoch, D., \& Fisher, T. (2015). Sense and "Sensitivity" Epistemic and Instrumental Approaches to Statistical Evidence. Stanford Law Review, 67, 557.

Enoch, D., Spectre, L., \& Fisher, T. (2012). Statistical Evidence, Sensitivity, and the Legal Value of Knowledge. Philosphy \& Public Affair, 40, 197 - 224.

Epstude, K., \& Roese, N. (2008). The functional theory of counterfactual thinking. Personality and Social Psychology Review, 12, 168 - 192.

Friedman, O., \& Turri, J. (2014). Is Probabilistic Evidene a Source of Knowledge? Cognitive Science, 39, 1062 1080. 
Gelman, A., Lee, D., \& Guo, J. (2015). Stan: a probabilistic programming language for Bayesian inference and optimization. Journal of Educational and Behavioral Statistics, 40, 530-543.

Halpern, J., \& Hitchcock, C. (2015). Graded Causation and Defaults. British Journal for the Philosophy of Science, 66, 413-457.

Hawthorne, J. (2003). Knowledge and Lotteries. New York, NY: Oxford University Press.

Ichikawa, J. (2018). Quantifiers, Knowledge, and Counterfactuals. Philosophy and Phenomenological Research, 82, 287 - 313.

Kruschke, J., \& Liddell, T. (2018). The Bayesian New Statistics: Hypothesis testing, estimation, metaanalysis, and power analysis from a Bayesian perspective. Psychonomic Bulletin \& Review, 25, 178-206.

Malle, B., Guglielmo, S., \& Monroe, A. (2016). A Theory of Blame. Psychological Inquiry, 25, 1 - 40.

Markman, K., McMullen, M., \& Elizaga, R. (2008). Counterfactual thinking, persistence, and performance: A test of the Reflection and Evaluation Model. Journal of Experimental Social Psychology, 44, 421 - 428.

McElreath, R. (2016). Statistical Rethinking: A Bayesian Course with Examples in $R$ and Stan. Boca Raton, FL: CRC Press.

Miller, A., Markman, K., Wagner, M., \& Hunt, A. N. (2013). Mental simulation and sexual prejudice reduction: the debiasing role of counterfactual thinking. Journal of Applied Social Psychology, 43, 190-194.

Niedermeier, K., Horowitz, I., \& Kerr, N. (1999). Informing Jurors of Their Nullification Power: A Route to a Just Verdict or Judicial Chaos? Law and Human Behavior, 23, 331-351.

Nozick, R. (1981). Philosophical Explanations. Cambridge, MA: Harvard University Press.

Pieters, R., \& Zeelenberg, M. (2005). On bad decisions and deciding badly: when intention-behavior inconsistency is regrettable. Organizational Behavior and Human Decision Processes, 97, 18-30.

Roese, N., \& Olson, J. (1997). Counterfactual Thinking: The Intersection of Affect and Function. Advances in Experimental Social Psychology, 29, 1-59.

Roese, N., \& Olson, J. (2012). Learning from yesterday's mistakes to fix tomorrow's problems: when functional counterfactual thinking and psychological distance collide. European Jouranal of Social Psychology, 42, 383-390.

Roush, S. (2005). Tracking Truth: Knowledge, Evidence, and Science. New York, NY: Oxford University Press.

Smallman, R., \& Roese, N. (2009). Counterfactual thinking facilitates behavioral intentions. Journal of Experimental Social Psychology, 45, 845-852.

Sykes, D., \& Johnson, J. (1999). Probabilistic Evidence Versus the Representation of an Event: The Curious Case of Mrs. Prob's Dog. Basic and Applied Social Psychology, 21, 199-212.
Tobia, M., Guo, R., Schwarze, U., Boehmer, W., G(ä)sher, J., Finckh, B., ... Sommer, T. (2014). Neural systems for choice and valuation with counterfactual learning signals. Neuroimage, 89, 57-69.

Wells, G. (1992). Naked Statistical Evidence of Liability: Is Subjective Probability Enough? Journal of Personality and Social Psychology, 62, 739-752.

Williamson, T. (2000). Knowledge and its Limits. New York, NY: Oxford University Press. 